\title{
Innovación didáctica para la formación de profesorado
}

\author{
SILVIA UMPIÉRREZ OROŃO** \\ Instituto de Formación Docente de San José - Uruguay \\ DELMA CABRERA ABREU ${ }^{* * *}$ \\ Instituto de Formación Docente de San José - Uruguay \\ PAOLA BRUCCOLERI ARRAMBIDE ${ }^{* * * *}$ \\ Instituto de Formación Docente de San José - Uruguay \\ Recibido el 29-04-20; primera evaluación el 03-06-21; \\ segunda evaluación el 23-06-21; aceptado el 21-07-21
}

\section{Resumen}

El trabajo valora una innovación con TIC en la práctica preprofesional en formación docente. Se organizaron seis instancias cuasiexperimentales en las que formadores de didáctica con estudiantes, realizaron análisis y reflexión sobre unidades fílmicas $360^{\circ}$. Se llevaron adelante tres observaciones no participantes sobre dichas instancias, encuestas $(n=39)$, siete entrevistas semiestructuradas y estudio documental de seis producciones de los participantes (docentes y estudiantes). Estos, valoraron los dispositivos (unidades fílmicas) por constituir un ambiente de aprendizaje de bajo riesgo, propiciar la colaboración entre los estudiantes y adaptarse a sus tiempos disponibles. Los dispositivos generados constituyen una

\footnotetext{
* Este trabajo fue apoyado por la Agencia Nacional de Investigación e Innovación y el Consejo de Formación en Educación, de Uruguay.

** Licenciada en Ciencias Biológicas por la Universidad de la República, máster y doctora en Educación por la Universidad ORT, Uruguay. Directora del Instituto de Formación Docente de San José, del Consejo de Formación en Educación, de la Administración Nacional de Educación Pública, Uruguay. https://orcid.org/0000-0003-3341-9687. Correo electrónico: sumpierrez16@gmail.com

*** Profesora de Biología, Magíster en Enseñanza de las Ciencias en Educación Media por CLAEH y en Educación, Sociedad y Política por FLACSO. Directora y docente efectiva del Instituto de Formación Docente de San José y del Profesorado Semipresencial, del Consejo de Formación en Educación, de la Administración Nacional de Educación Pública, Uruguay. https://orcid.org/0000-0002-8506-4489. Correo electrónico: delmacabrera@gmail.com

**** Analista programadora, egresada de la Universidad de la República y tester de software; ha realizado especializaciones y cursos relacionados con las tecnologías digitales y la educación. Docente del Instituto de Formación Docente de San José, del Consejo de Formación en Educación, de la Administración Nacional de Educación Pública, Uruguay. https://orcid.org/0000-0003-1012-8000. Correo electrónico: pbruccoleri@gmail.com
} 
herramienta de aprendizaje que adquiere valor más por la intervención didáctica que se realiza con ellos, que por sus características técnicas.

Palabras clave: innovaciones educativas, formación de profesores, didáctica, tecnologías de la información y la comunicación.

\section{Didactic innovation in teachers training}

\section{Abstract}

The work values an innovation with ICT in pre-professional practice in teacher training. Six quasi-experimental instances were organized in which didactic trainers with students carried out analysis and reflection on $360^{\circ}$ film units. Three non-participant observations were carried out on these instances, surveys $(\mathrm{n}=$ 39), seven semi-structured interviews and a documentary study of six productions by the participants (teachers and students). They valued the devices (film units) for constituting a low-risk learning environment, fostering collaboration among students, and adapting to their available times. The devices generated constitute a learning tool that acquires value more for the didactic intervention that is carried out with them, than for their technical characteristics.

Keywords: educational innovations, teacher training, didactics, information and communication technologies.

\section{Inovaçáo no ensino para treinamento de professorado ${ }^{\circ}$}

\section{Resumo}

O trabalho valoriza uma inovação com as TIC na prática pré-profissional na formação de professores. Foram organizadas seis instâncias quase-experimentais nas quais formadores didáticos com alunos realizaram análises e reflexôes em unidades de filme $360^{\circ}$. Nessas instâncias foram realizadas três observaçôes não participantes, questionários $(\mathrm{n}=39)$, sete entrevistas semiestruturadas e um estudo documental de seis produçóes dos participantes (professores e alunos). Eles valorizaram os dispositivos (unidades de filme) por constituírem um ambiente de aprendizagem de baixo risco, fomentando a colaboraçáo entre os alunos e adaptando-se aos horários disponíveis. Os dispositivos gerados constituem uma ferramenta de aprendizagem que adquire valor mais pela intervenção didática que se realiza com eles do que pelas suas características técnicas.

Palavras-chave: inovaçôes educacionais, formação de professores, didática, tecnologias da informação e comunicação. 


\section{INTRODUCCIÓN}

Con frecuencia las innovaciones educativas no siguen un camino que incluya su valoración, monitoreo y seguimiento o son valoradas posteriormente a su implementación (Umpiérrez y Rodríguez, 2017). En este trabajo se propone realizar dicha valoración previamente, como forma de propiciar su adopción, dado que una de las dificultades identificadas en la transformación y el cambio educativo es la resistencia de los docentes. Esta resistencia está fundamentada principalmente por la necesidad de confiabilidad en la posibilidad de mejora que acompaña la nueva propuesta a ser adoptada en el aula. Incorporar innovaciones en las prácticas de enseñanza requiere energía, recursos y tiempos. Además, si el sistema educativo invierte en equipamiento para una innovación y esta no da resultados de mejora, se resiente la posibilidad de considerar valiosa la inversión en propuestas que apunten a la transformación y el cambio (Umpiérrez y Rodríguez, 2017).

El presente trabajo presenta la valoración de una propuesta de innovación con tecnologías de la información y la comunicación (TIC) en las actividades de la práctica preprofesional, en la formación de profesores de educación media de Uruguay. Se utiliza un escenario cuasiexperimental, por medio del uso de videos de actividades de enseńanza en educación media en $360^{\circ}$. Se sitúa a docentes y estudiantes de profesorado de educación media en situación de uso de la innovación, proponiéndoles la observación y el análisis didáctico de dichos materiales. De esta manera, se pretendió profundizar en las dimensiones didáctica y tecnológica de los dispositivos en prueba: conocer cuáles contenidos pudieron ser abordados en la aplicación, cómo se hizo, qué aportes formativos se promovieron y qué aspectos de los dispositivos fueron más apreciados o, por el contrario, requieren ser mejorados, de acuerdo con la percepción de los participantes.

Los objetivos del proyecto en el marco del cual se desarrolla este trabajo incluyen enriquecer y diversificar la formación de profesores, al incorporar y valorar modalidades innovadoras que propician el trabajo en la virtualidad, asincrónico, interdisciplinar y grupal, utilizando actividades de enseñanza filmadas en $360^{\circ}$ en centros de educación media, como unidades de reflexión didáctica para la formación del profesorado. La investigación buscó recoger las percepciones de los usuarios (docentes y estudiantes de formación docente) cuando aplican dichas modalidades, en el análisis didáctico de actividades de enseñanza, en la práctica preprofesional de esa formación. Las preguntas que se plantean responder son: ¿qué contenidos pueden ser abordados al utilizar las filmaciones en prueba?; ¡cómo se abordan?; ¡̇en qué aspectos formativos 
muestran tener potencial de aporte?; ¿qué cuestiones valoran los participantes que las utilizaron?; ¿qué elementos perciben como pasibles de mejora o podrían ser limitantes de su uso?

El trabajo se llevó adelante en el contexto de la formación inicial de docentes de educación media de Uruguay. Los formatos de trabajo que se propusieron valorar resultan disruptivos en este país, debido a que la formación práctica preprofesional normalmente se lleva adelante mediante el análisis de actividades de enseñanza observadas presencialmente. La mejora que se pretende a través de la innovación que se está presentando aquí, se encamina a ofrecer alternativas formativas para el perfil de los estudiantes uruguayos, muchos de los cuales deben viajar para realizar actividades presenciales, trabajan o tienen obligaciones familiares bajo su responsabilidad, como son las tareas de cuidado y crianza (CFE, 2015).

En Uruguay la formación práctica preprofesional además se realiza desde una única disciplina, que corresponde con la especialidad para la que se está formado para enseñar el estudiante (biología, matemática, inglés, etc.) e individualmente. Los dispositivos innovadores en prueba ofrecen la posibilidad de trabajo interdisciplinario y grupal. Estas dos características adhieren al desarrollo de competencias para el trabajo colaborativo, elemento identificado por Vaillant (2019) como uno de los facilitadores de la innovación.

\section{Marco teórico}

Este trabajo se desarrolla en el campo de la innovación pedagógica. De acuerdo con la concepción ofrecida por Aguerrondo (2014), esta refiere a propuestas que alteran lo didáctico y los recursos, aspectos estructurales de la escuela. Para Umpiérrez y Rodríguez (2017), la innovación comienza con la identificación de un problema educativo, y sigue un itinerario que debe incluir, al inicio, la presentación ante pares, previo a ser expandida a otras comunidades educativas. Esa presentación constituye parte de la validación y se realiza en la etapa de gestación de la innovación. La validación proporciona múltiples elementos: monitorea los avances, proporciona orientaciones de mejora, y ofrece más confiabilidad a quien aspire a adoptarla.

La difusión de innovaciones educativas no está garantizada por la existencia de iniciativas personales o dentro de un aula específica. Deben seguir líneas claras que respondan a la necesidad del sistema educativo: «Concebir la innovación como una oportunidad de explorar la responsabilidad de la academia hacia el contexto en el que está inserta» (González y Cruzat, 2019, p. 119). 
El contexto de la innovación que se presenta en este trabajo es la formación práctica en didáctica de la formación de profesores. Promover el cambio requiere mucha energía porque implica modificar la cultura institucional (Aguerrondo y Tiramonti, 2016). No obstante, se hace necesario que los docentes se preparen acompañando las transformaciones del mundo sin perder la visión y el interés por las locales (Vaillant, 2019); ello incluye adoptar el uso de TIC en las prácticas de enseñanza. Incorporarlas como herramientas pedagógicas en su formación, los hace proclives a utilizarlas luego productivamente en sus propuestas de enseñanza (Fernández, 2016). Afirma Vaillant (2019): «sabido es que lo que hace un futuro docente no es tanto lo que le dicen los libros de pedagogía, sino replicar el modelo que tiene frente» (p. 6).

Para que la innovación en la formación de los profesores tenga alcance en los procesos de cambio, Vaillant (2019) proporciona, entre otras, dos dimensiones que deben ser abordadas: la metodológica y las actividades de aprendizaje. Lo metodológico, incluye alternar, no sustituir, la "modalidad clásica» (Vaillant, 2019 , p. 5), propender a la conformación de comunidades de práctica, sin excluir las instancias individuales, e incorporar la virtualidad. Las actividades de aprendizaje se refieren a la forma en que se diseñan e implementan las prácticas con el apoyo de recursos pedagógicos, en el caso de esta investigación, los tecnológicos.

Los aprendizajes en torno a cómo enseñar, tienen una importancia medular en la formación de docentes. Siguiendo con las categorías de Vaillant (2019):

- lo metodológico es «la manera en la que la preparación del profesorado se implementa» (Vaillant, 2019, p. 5) y donde, debido al surgimiento continuo de nuevas tecnologías, se encontrarán la mayor cantidad de innovaciones.

- las actividades de aprendizaje son las acciones concretas que el docente, en el aula, lleva adelante para enseñar determinados contenidos o desarrollar ciertas competencias, apoyándose en recursos didácticos.

Las TIC pueden constituirse en herramientas didácticas que, en la formación de docentes, aporten a la autogestión de los estudiantes (Landín, 2015). Les mejora la posibilidad de organización de sus tiempos, recursos, espacios, «nuevas formas de interacción social» (Landín, 2015, p. 125), y aprendizaje colaborativo. El uso de TIC no excluye la actividad presencial, sino que diversifica los escenarios de aprendizaje. La posibilidad de acceder a la realidad que se pretende estudiar, sin desplazarse físicamente, pero a la vez manteniendo el contacto con otros, es una potencialidad del uso de actividades de enseñanza filmadas que se desea evidenciar a través de esta investigación. 
Existen numerosas experiencias que han trabajado en ese mismo sentido, como Fernández y Fernández (1994), Fernández, Elórtegui y Medina (2003), Anijovich y Cappelletti (2018). Estos trabajos citados recuperan dentro de otros valores, la posibilidad de observar y analizar situaciones reales, pero en un ambiente seguro y controlado. Sin embargo, no se han identificado investigaciones que trabajen con filmaciones en $360^{\circ}$ en la formación superior.

Los dispositivos que incluyen filmación en $360^{\circ}$ se integran a propuestas de realidad virtual, si bien esta última incorpora mayor cantidad de recursos. La observación de material filmado en $360^{\circ}$ contribuye a la formación de imágenes mentales, una representación perceptiva de la información no verbal en la memoria, que puede ser estimulante (Bogicevic, Seo, Kandampully, Liu y Ruddet, 2019).

Se ha reportado el uso de $360^{\circ}$ en ciencias aplicadas de la computación, en medicina, deportes, periodismo, conservación ambiental y recreación. La producción de estos materiales se ha relacionado con la democratización en el acceso al mundo y al conocimiento, al ampliar el acceso socio cultural a determinados procesos o fenómenos y al hacerlo de forma asistida e inclusiva para personas con diferentes capacidades. Pueden ser utilizados con fines educativos e investigativos (De Fátima, Pedreira da Silva y Ferreira da Costa, 2020).

De acuerdo con De Fátima et al. (2020), la usabilidad es un concepto utilizado formalmente al evaluar la capacidad de un dispositivo para alcanzar los objetivos del usuario y satisfacer sus necesidades, en un determinado contexto. Pruebas realizadas por los autores con estas tecnologías, muestran que deben ser considerados como elementos de valoración de usabilidad:

- Tipo de usuario: ¿qué personas pueden interactuar con el dispositivo?

- Contexto de uso: ¿dónde se sitúan o de dónde provienen dichas personas?

- Eficacia: consecución de los objetivos del usuario, utilizando el dispositivo.

- Eficiencia: relación entre los objetivos alcanzados por el usuario y el costo involucrado.

- Satisfacción: evidencias sobre el confort y aceptación en el uso del producto por parte del usuario.

Algunas de las limitaciones reportadas son: la observación de videos en $360^{\circ}$ permiten la formación de imágenes mentales, pero no la sensación de presencia; a veces se desarrollan en escenarios hipotéticos; los resultados están influidos por la subjetividad de las personas, como por ejemplo su procedencia 
cultural, y no siempre se logra una óptima calidad de las imágenes (Bogicevic et al., 2019) lo que puede disminuir su verosimilitud.

\section{Metodología}

Se trata de una investigación enmarcada metodológicamente en el paradigma cualitativo, con finalidades que aportan a la transformación y el cambio, por lo que puede definirse como un estudio crítico (Paz Sandín, 2003). Se aborda el análisis a partir de datos que surgen de casos instrumentales, tratándose de un muestreo intencional. Los hallazgos describen, interpretan e intentan comprender en profundidad una serie de particularidades sobre una innovación educativa, que no son generalizables, pero contribuyen al conocimiento del fenómeno en estudio (Hernández, Fernández y Baptista, 2010). Se trabaja entonces, con generalizaciones a la interna de la muestra.

La fase previa consistió en elaborar unidades fílmicas en $360^{\circ}$ y protocolos. Cada unidad fílmica contiene un fragmento de actividades de enseńanza a nivel de educación media. Estas actividades de enseñanza fueron no modélicas, es decir que no se prepararon especialmente para ser grabadas, sino que son una práctica de enseńanza cotidiana, de profesores que estuvieron dispuestos a colaborar voluntariamente con el proyecto. Se logró una diversidad de unidades fílmicas que incluyó actividades de enseñanza de niveles tanto medio básico (12-15 años) como superior (15-18 años), en aula común, en laboratorio, en el campo y hasta en los pasillos de una institución de educación media. Por su parte, los protocolos orientan la reflexión y el análisis didáctico que se puede realizar observando dichas unidades fílmicas en el marco de la asignatura didáctica de la formación de profesorado de educación media. Los protocolos acompañan el trabajo de forma opcional; un formador puede optar por usar solo la unidad fílmica para realizar observación, análisis y reflexión sobre lo que sucede en las actividades de enseñanza que se muestran en ella, o hacerlo con el apoyo de protocolo como guía de clase.

A continuación, se organizaron instancias cuasiexperimentales, consistentes cada una de ellas, en una clase de didáctica curricular, parte del curso normal de la formación de profesorado para educación media, en la cual se introduce como elemento disruptivo el uso de los recursos digitales que se pretende valorar el equipo de investigación: unidades fílmicas y protocolos. En cada instancia cuasiexperimental, formadores de didáctica con sus estudiantes, realizaron observación, análisis y reflexión de unidades fílmicas en $360^{\circ}$. La unidad fílmica que se dispuso para cada instancia cuasiexperimental fue seleccionada por el equipo del proyecto en diálogo con los docentes de 
didáctica. La adjudicación de la categoría «cuasiexperimental», entonces, deriva de que se trata de un hecho que pertenece a la vida educativa natural de las personas (docentes y estudiantes observando y analizando una actividad de enseñanza) donde se introduce un elemento disruptivo, pero no se controlan todas las variables (Hernández et al., 2010). Si bien la cuasi-experimentación proviene del paradigma positivista, en este caso los resultados no son cuantificables, no se utilizarán para predecir, explicar, generalizar o modelizar un proceso sino para comprender de qué forma los participantes se vinculan con las diferentes características y potencialidades de una innovación.

Cada instancia cuasiexperimental es una unidad de análisis, ya que se constituye en caso de estudio al incorporarse en torno a cada instancia, múltiples fuentes de datos (observación no participante, estudio de documentos, entrevistas y encuestas), para realizar la valoración de los dispositivos. Se llevó a cabo observación no participante de algunas de las instancias, de acuerdo con la factibilidad. Se realizó análisis de contenido de documentos tales como intercambios por los foros y materiales producidos por los docentes y estudiantes, de las seis instancias. Además, se llevaron a cabo entrevistas semiestructuradas, a docentes y estudiantes, donde se abordaron detalles de la actividad planteada con los dispositivos, los resultados obtenidos, sus potencialidades, posibles aplicaciones y sus debilidades. Finalmente se aplicó a docentes y estudiantes una encuesta donde se recabaron datos sobre su nivel de formación, especialidad, institución formadora, modalidad de la carrera, su percepción de los dispositivos y su utilización.

El análisis permitió sistematizar los datos sobre la dimensión didáctica en dos categorías reformuladas a partir de la propuesta de trabajo de Vaillant (2019): metodología y actividades de aprendizaje. Para la primera, el análisis construye conocimiento en torno a la diversidad de formas en que los docentes plantearon la actividad con el dispositivo: presencial/virtual; sincrónico/asincrónico; individual/grupal; disciplinar/interdisciplinar. En la segunda, los datos permitieron conocer y describir los tipos de contenidos y las estrategias que los dispositivos en prueba permitieron abordar y desplegar.

Para la dimensión tecnológica se utilizaron cinco categorías, tomadas del trabajo de De Fátima et al. (2020), de usabilidad del dispositivo. Sobre el tipo de usuario, se buscaron evidencias sobre los niveles formativos que admite el uso de la innovación. Sobre el contexto de uso, se reportó la procedencia institucional de los docentes y estudiantes que utilizaron los dispositivos en prueba. La eficacia se valoró a través de las percepciones de los participantes sobre la capacidad que muestra la innovación para llevar adelante con ella, las tareas de la clase de didáctica. La eficiencia se visualiza en la descripción de los materiales 
y entornos que fueron requeridos para ello. Por último, la satisfacción se logró valorar recogiendo evidencias sobre las apreciaciones de los usuarios sobre las potencialidades y posibles mejoras que detectan en los dispositivos.

Cabe aclarar que las fuentes de los hallazgos que aquí se reportan provienen por un lado de la observación no participante, que de acuerdo con De Fátima et al. (2020) es una fuente objetiva, y por otro, de las apreciaciones y opiniones vertidas por los participantes en lo recogido con el resto de las técnicas, que son datos subjetivos, es decir, provienen de la percepción de los usuarios.

\section{Resultados}

Se implementaron seis instancias cuasiexperimentales, para la observación, análisis y reflexión de unidades fílmicas en $360^{\circ}$. Algunos detalles de los mismos se detallan en la Tabla 1.

Las unidades fueron utilizadas por docentes de cinco especialidades: biología, inglés, matemática, idioma español y literatura.

Tabla 1. Características de las instancias cuasiexperimentales

\begin{tabular}{|c|c|c|c|c|c|}
\hline Caso & $\begin{array}{l}\text { Número } \\
\text { de estu- } \\
\text { diantes }\end{array}$ & $\begin{array}{l}\text { Número } \\
\text { de do- } \\
\text { centes }\end{array}$ & $\begin{array}{l}\text { Especialidad y nivel } \\
\text { en que enseñan los } \\
\text { docentes }\end{array}$ & $\begin{array}{l}\text { Unidad } \\
\text { fílmica } \\
\text { utilizada }\end{array}$ & Protocolo utilizado \\
\hline 1 & 8 & 1 & $\begin{array}{l}\text { Didáctica I de Inglés } \\
\left(2^{\circ} \text { año de formación }\right. \\
\text { preprofesional) }\end{array}$ & $\begin{array}{l}\text { Clase de } \\
\text { inglés }\end{array}$ & Diseño del formador \\
\hline 2 & 4 & 1 & $\begin{array}{l}\text { Didáctica I de Mate- } \\
\text { mática ( } 2^{\circ} \text { año de for- } \\
\text { mación preprofesional) }\end{array}$ & $\begin{array}{l}\text { Clase de } \\
\text { matemá- } \\
\text { tica }\end{array}$ & $\begin{array}{l}\text { Proporcionado por el } \\
\text { equipo de investiga- } \\
\text { ción, adaptado por el } \\
\text { formador }\end{array}$ \\
\hline 3 & 8 & 1 & $\begin{array}{l}\text { Didáctica II de Biolo- } \\
\text { gía }\left(3^{\circ} \text { año de forma- }\right. \\
\text { ción preprofesional })\end{array}$ & $\begin{array}{l}\text { Clase de } \\
\text { biología }\end{array}$ & Diseño del formador \\
\hline 4 & 15 & 3 & $\begin{array}{l}\text { Didáctica III de Inglés, } \\
\text { Matemática y Biología } \\
\text { ( } 4^{\circ} \text { ańo de formación } \\
\text { preprofesional) }\end{array}$ & $\begin{array}{l}\text { Clase de } \\
\text { biología }\end{array}$ & $\begin{array}{l}\text { Diseńo de los forma- } \\
\text { dores }\end{array}$ \\
\hline 5 & 6 & 1 & $\begin{array}{l}\text { Didáctica II de Litera- } \\
\text { tura }\left(3^{\circ} \text { año de forma- }\right. \\
\text { ción preprofesional) }\end{array}$ & $\begin{array}{l}\text { Clase de } \\
\text { literatura }\end{array}$ & $\begin{array}{l}\text { Proporcionado por } \\
\text { el equipo de investi- } \\
\text { gación }\end{array}$ \\
\hline
\end{tabular}




\begin{tabular}{cccccc}
\hline Caso & $\begin{array}{c}\text { Número } \\
\text { de estu- } \\
\text { diantes }\end{array}$ & $\begin{array}{c}\text { Número } \\
\text { de do- } \\
\text { centes }\end{array}$ & $\begin{array}{c}\text { Especialidad y nivel } \\
\text { en que enseñan los } \\
\text { docentes }\end{array}$ & $\begin{array}{c}\text { Unidad } \\
\text { fílmica } \\
\text { utilizada }\end{array}$ & Protocolo utilizado \\
\hline 6 & 20 & 1 & $\begin{array}{l}\text { Introducción a la di- } \\
\text { dáctica Idioma Español } \\
\left(1^{\circ} \text { ańo de formación }\right. \\
\text { preprofesional })\end{array}$ & $\begin{array}{l}\text { Clase de } \\
\text { filosofía }\end{array}$ & $\begin{array}{l}\text { Proporcionado por } \\
\text { el equipo de investi- } \\
\text { gación }\end{array}$ \\
\hline
\end{tabular}

Fuente: datos tomados de la observación no participante, estudio documental y entrevistas semiestructuradas.

Se llevó a cabo observación no participante en tres de las seis instancias. Para la construcción de los seis casos de estudio en torno a las instancias cuasiexperimentales, se realizó análisis de contenido de intercambios en dos foros y cuatro conjuntos de materiales producidos por los docentes y estudiantes, pertenecientes a las seis instancias. Se realizaron siete entrevistas semiestructuradas, tres a estudiantes y cuatro a docentes y se aplicó a docentes y estudiantes $(\mathrm{N}=69)$, una encuesta que fue respondida por 32 estudiantes y 7 docentes.

Ninguno de los casos presentó imposibilidad de llevar adelante la observación, el análisis y la reflexión con los materiales proporcionados (la unidad fílmica y, en algunos, los protocolos guías). Si bien en cada caso se los utilizó de diferente manera, con diferente profundidad, todos los actores manifestaron una opinión favorable del instrumento de aprendizaje que estaban experimentando: «Los estudiantes miraron las unidades fílmicas más de una vez y se habían realizado intercambios entre ellos previo a la clase...era la última clase del año... Estaban cansados, tenían otro parcial al día siguiente» (entrevista docente del caso 5). Las observaciones no participantes permitieron ver a docentes y estudiantes motivados con este, interactuando con escasas dificultades de índole pedagógica y tecnológica. Las percepciones de los participantes, recogidas por las otras dos técnicas (análisis de contenido y encuestas), proporcionan una visión personal detallada y valiosa tanto de las potencialidades como de las debilidades de los dispositivos.

\subsection{La dimensión didáctica}

La categoría «metodología» permitió describir las modalidades en que los docentes desarrollaron la observación, el análisis didáctico y la reflexión, con las unidades fílmicas. $\mathrm{Al}$ analizar las alternativas presencial/virtual, sincrónico/ asincrónico, individual/grupal y disciplinar/interdisciplinar, se observa que todos los equipos realizaron combinaciones de dichas alternativas, en diferentes momentos del proceso. Dicho resultado se sintetiza en la Tabla 2. En esta, 
se han colocado en las cuatro primeras filas las metodologías más tradicionales, y en las cuatro siguientes las menos tradicionales. Todos los equipos combinaron modalidades de ambos grupos; los casos 2 y 4 son los que usaron con mayor intensidad las del segundo grupo.

Por otra parte, se han sintetizado los hallazgos que nutrieron la categoría "Actividades de aprendizaje», referida a los tipos de contenidos y las estrategias que los dispositivos en prueba permitieron abordar y desplegar, en la Figura 1. Los contenidos fueron agrupados en cuatro tipos, los cuales se presentan en los siguientes párrafos.

Tabla 2. Metodologías usadas en la aplicación de cada caso

\begin{tabular}{|c|c|c|c|c|c|c|}
\hline CASOS & 1 & 2 & 3 & 4 & 5 & 6 \\
\hline Presencial & & & & & & \\
\hline Sincrónica & & & & & & \\
\hline Individual & & & & & & \\
\hline Disciplinar & & & & & & \\
\hline Virtual & & & & & & \\
\hline Asincrónica & & & & & & \\
\hline Grupal & & & & & & \\
\hline Interdisciplinar & & & & & & \\
\hline
\end{tabular}


Figura 1. Tipos de contenidos que se pudieron trabajar durante las clases de didáctica en las que se utilizaron las unidades fílmicas y la frecuencia relativa de cada uno.

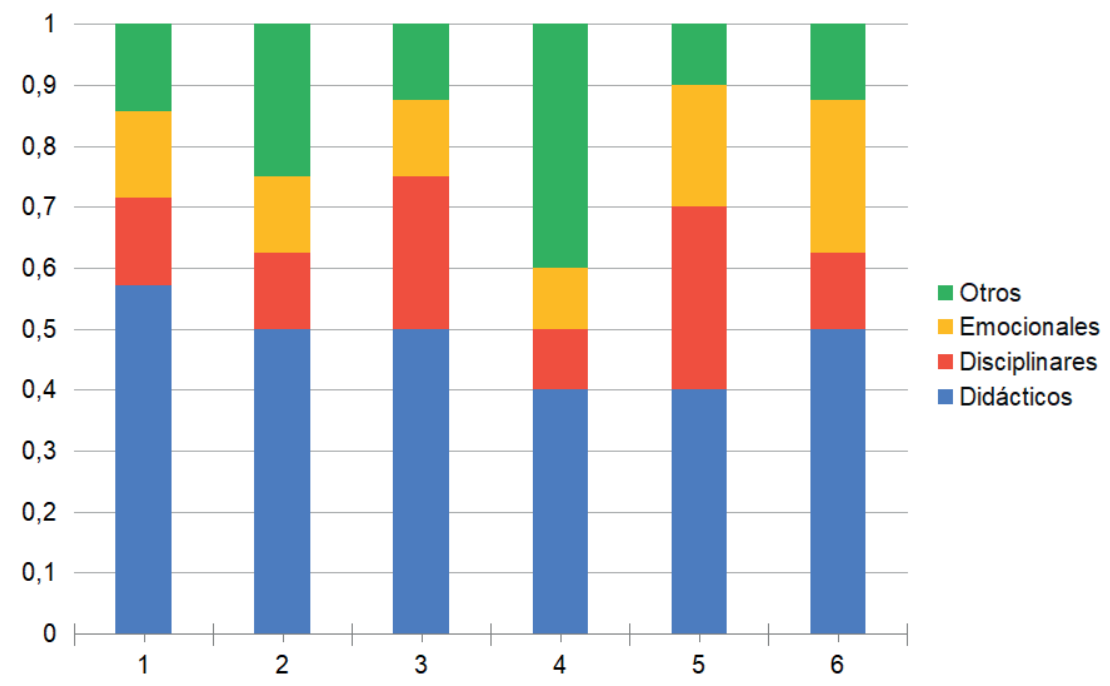

Fuente: observación no participante, entrevistas semiestructuradas y estudio documental.

Se presentan ejemplos de los hallazgos recogidos (ver al final de la sección, Tabla 3) y se evidencian las estrategias utilizadas.

El tipo «didáctico» se refiere al análisis de lo que el docente de la unidad fílmica hace y a qué recurre para lograr enseñar. En el caso 1, la consigna indicaba focalizar el análisis de la actividad de enseńanza observada en la unidad fílmica en: manejo del aula, objetivos de aprendizaje y secuencia de actividades. Además, les proporcionó un listado de preguntas, que orientaban la observación en torno a: ideas disciplinares, objetivos, ejercicios, tareas o problemas propuestos e interacciones entre quienes participan de la actividad de enseñanza observada.

El tipo «disciplinar» reúne los contenidos que provienen de uno o varios campos del conocimiento de la o las disciplinas de las especialidades a que pertenecen los estudiantes participantes. El caso 5 fue el que desarrolló análisis en torno a este tipo de contenidos en similar proporción que el didáctico. En esta instancia cuasiexperimental, la docente envía al grupo de estudiantes la unidad fílmica con el protocolo "Incidente crítico 2» que guiaba el análisis, proporcionado por el equipo, que puede consultarse en http://recyreap. cfe.edu.uy/. 
El contenido etiquetado con el tipo «emocionales», hace referencia a analizar los vínculos e interacciones en la unidad fílmica, y a los aspectos autobiográficos, a la reminiscencia provocada por la unidad fílmica, a la historia escolar o experiencias vividas en otros contextos. En el caso 6, donde se presentó mayor frecuencia relativa del tipo «emocional» con relación a los otros casos (Ver Figura 1), se observa dentro de la consigna que presenta la docente de didáctica el siguiente texto: «Reflexionamos acerca de la conjugación de lo humano y lo material en una clase y su importancia en los procesos de enseñanza y el aprendizaje».

Dentro del tipo de contenidos catalogados como «otros» quedó consignado el análisis de aspectos críticos (sociales, contextuales, políticos), tecnológicos y epistemológicos. En el caso 2 se encontraron evidencias relativamente importantes, sobre aspectos sociales y contextuales. En el caso 4, por su parte, los contenidos giraron en torno al uso de tecnologías digitales que hacía el profesor de la unidad fílmica. En el caso 3 aparecen elementos en el análisis que hacen alusión a la construcción y aplicación del conocimiento. En la tabla 3 se transcriben evidencias de cada tipo de contenido.

Tabla 3. Evidencias de los contenidos abordados.

\begin{tabular}{|c|c|c|}
\hline Tipo de contenido & Evidencias & Fuente \\
\hline Didáctico & $\begin{array}{l}\text { "Yo miré mucho a la profesora.» } \\
\text { «Me siento más consciente de lo } \\
\text { que hago en mis clases.» } \\
\text { "Aprender a observar y a } \\
\text { observarme.» }\end{array}$ & $\begin{array}{l}\text { Caso } 1 \\
\text { Observación no participante. } \\
\text { Frases vertidas por los } \\
\text { estudiantes durante la } \\
\text { instancia cuasiexperimental }\end{array}$ \\
\hline Disciplinar & $\begin{array}{l}\text { «Discutir sobre cómo integrar lo } \\
\text { semántico, o sea lo que decía el } \\
\text { texto con la forma del texto que } \\
\text { estaba trabajando el profesor, les } \\
\text { iba a llevar manejar conocimientos } \\
\text { específicos del autor y del texto.» }\end{array}$ & $\begin{array}{l}\text { Casi } 5 \\
\text { Entrevista semiestructurada a } \\
\text { la docente }\end{array}$ \\
\hline Emocionales & $\begin{array}{l}\text { «Pizarrón viejo y querido: una } \\
\text { construcción colaborativa de } \\
\text { enseñanza y aprendizaje». }\end{array}$ & $\begin{array}{l}\text { Caso } 6 \\
\text { Observación no participante. } \\
\text { Frase escrita en el pizarrón. }\end{array}$ \\
\hline
\end{tabular}

Fuente: observación no participante, entrevistas y estudio documental. 


\begin{tabular}{|c|c|c|}
\hline Tipo de contenido & Evidencias & Fuente \\
\hline $\begin{array}{l}\text { Sociales y } \\
\text { contextuales }\end{array}$ & $\begin{array}{l}\text { «La práctica no siempre es en } \\
\text { parejas y luego del egreso son pocas } \\
\text { las oportunidades de ir a visitar las } \\
\text { clases de un colega en grupo. La } \\
\text { diferencia de esta observación es } \\
\text { que se puede compartir con todo el } \\
\text { que quiera sumarse....» }\end{array}$ & $\begin{array}{l}\text { Caso } 2 \\
\text { Foro en plataforma. Texto } \\
\text { escrito por la docente de } \\
\text { didáctica. }\end{array}$ \\
\hline Tecnológicos & $\begin{array}{l}\text { "Analizar qué competencias } \\
\text { digitales despliega el formador en } \\
\text { la unidad fílmica y presentar un } \\
\text { recurso tecnológico con sugerencias } \\
\text { de mejora para la profesora que } \\
\text { aparece en ella.» }\end{array}$ & $\begin{array}{l}\text { Caso } 4 \\
\text { Observación no participante. } \\
\text { Parte de la consigna dada a } \\
\text { los estudiantes. }\end{array}$ \\
\hline
\end{tabular}

Fuente: observación no participante, entrevistas y estudio documental.

\subsection{La dimensión tecnológica}

Se presentan a continuación las evidencias correspondientes a las cinco categorías definidas para valorar el dispositivo innovador con relación a su usabilidad.

Para «Tipo de usuario», se remitió a identificar los niveles formativos en que mostró poder aplicarse la innovación. La aplicación fue posible en todos los niveles pre profesionales en didáctica, a lo largo de los cuatro años. Además, los participantes afirmaron que puede ser utilizado en formación permanente de docentes egresados. La docente de didáctica del caso 1, en la entrevista, manifestó: "Cuando trabajas con docentes egresados se puede trabajar con un coach o supervisor que no necesariamente sea un profesor de didáctica». Dos estudiantes del caso 2, en el foro del aula en plataforma sobre el que se realizó análisis de contenido, opinan: «... más allá de poder herir algunos egos, esas video clases pueden servir de mucho para el aprendizaje de los docentes que ya se recibieron».

Para «Contexto de uso», se registró la procedencia institucional y formativa de los docentes y estudiantes que utilizaron los dispositivos en prueba, en las diferentes modalidades de formación del país. Por medio de las seis instancias cuasiexperimentales, se logró incluir todo el espectro institucional de la oferta pública de formación docente del país. También se logró incluir a las dos modalidades en las que se lleva adelante: presencial y semipresencial. Los estudiantes pertenecían a un total de siete especialidades, de acuerdo con los datos de la encuesta. 
Sobre la categoría «Eficacia», se valoró la innovación a través de las percepciones de los participantes sobre la capacidad que muestra la innovación para llevar adelante con ella, las tareas de la clase de didáctica. Un estudiante del caso 2, en el foro analizado, afirma con relación a la realización de trabajo grupal habilitado por el uso de los instrumentos: «promover la reflexión y el diálogo... Dos cabezas piensan más que una». La docente del mismo caso, en la entrevista manifiesta: la tecnología $360^{\circ}$ permite observar «tanto en el pizarrón, como a los alumnos como a la docente, como un estar en la clase que no te da la cámara fija». En la encuesta, la fortaleza del análisis didáctico de actividades de enseñanza filmadas que más fue seleccionada por estudiantes es la que considera que "genera condiciones de acceso a la reflexión didáctica» (26 de 32) y "permite identificar adecuadamente los sucesos que ocurren en el aula» (19 de 32).

La categoría «Eficiencia» está referida a la descripción de los materiales y entornos que se requieren para el uso de la innovación. Durante las observaciones no participantes se vio a los estudiantes y docentes accediendo tanto desde equipos personales como proporcionados por la institución: celulares, tablets, computadoras. El entorno de trabajo requirió en algunos casos, según cómo cada docente hubiera planteado el desarrollo de la actividad, una pantalla para la observación grupal de las unidades fílmicas. En el caso 3, un estudiante entrevistado manifestó: «son herramientas con la que todos los estudiantes pueden contar en sus casas». Sin embargo, por otro lado, algunos estudiantes debieron acceder a la unidad fílmica en sus dispositivos personales, ya que la misma era bloqueada por la red pública disponible en forma libre en los centros de formación docente. En esos casos, los estudiantes debieron recurrir al uso de datos móviles propios. La docente del caso 3 en la entrevista manifiesta, con relación a los entornos necesarios, que esta innovación sortea la dificultad económica (pago de pasajes) que tienen los estudiantes de visitarse entre ellos para observación mutua de sus actividades de enseńanza. «Por medio de la filmación esto sería más accesible».

La categoría «Satisfacción» reúne las apreciaciones de los usuarios sobre las potencialidades y/o posibles mejoras que detectan en los dispositivos. El docente del caso 5, en la entrevista expresa:

"Además, al ser una unidad filmica de 11 minutos, se puede ver una y otra vez, y se trata de un segmento, seleccionado y focalizado. Eso permite mayor profundidad que si observaran una clase. Cuando observás una clase entera, tenés que recordar lo que pasó. Aqui, en la unidad fílmica, tenés 
disponible lo que pasó realmente, no tenés que recurrir a la memoria. A mí me parece una herramienta muy potente».

En el caso 2, durante los intercambios en foro, dos estudiantes mantienen el siguiente diálogo:

- "Este tipo de herramientas las veo muy apropiadas para utilizarlas en formación docente. Aunque la observación directa -presencial- aporta mucho, esta es una herramienta que nos brinda otras posibilidades».

- "Hola, M., estoy de acuerdo con lo que decís, de que es una herramienta muy apropiada para la formación docente, si bien la observación directa es favorable, pero a veces es casi imposible por un tema de espacio y de tiempo el poder acudir a todas ellas».

También se recogieron percepciones de las posibles mejoras a introducir en los dispositivos. En la encuesta, los estudiantes afirmaron que en la unidad fílmica no podían ver bien el pizarrón. La encuesta informa que 20 de los 32 estudiantes respondientes, eligieron como debilidad los aspectos técnicos (sonido, imagen). Otra posible limitación elegida en la misma frecuencia, fue que la presencia de la cámara altera el funcionamiento natural del aula (docentes y/o estudiantes). Sin embargo, la docente del caso 2 afirma en la entrevista, que no se evidencia, en las unidades fílmicas, incomodidad por parte de los alumnos cuando fueron filmados. Los resultados con relación a elegir esta innovación solo como una alternativa, cuando existe necesidad de andamiar los aprendizajes, fue marcada por 20 estudiantes en la encuesta. Veintiocho estudiantes, por su parte, la consideraron no una alternativa auxiliar, sino una opción disponible siempre dentro de las modalidades de aprendizaje didáctico.

Una observación destacable que surge de las entrevistas, es que los estudiantes y docentes encontraron, en la observación grupal de una práctica de enseñanza de un "desconocido», la oportunidad de expresarse en un ambiente de bajo riesgo, donde no se hiere el amor propio de ninguno, lo que les proporciona más libertad en el análisis y la reflexión. Tal como afirma la docente del caso 2, en la entrevista:

En Didáctica 3 curso presencial, ellos están muy susceptibles, uno va a visitar las clases, a hacerles la crítica. A ellos les duele lo que uno les dice, y están como a la defensiva, entonces poder mirar una clase objetivamente sin que sea mi clase, ver todo lo que podemos sacar, a ellos les da como que más elementos para ser más reflexivos con sus propias prácticas. 
A la vez le otorga la oportunidad al docente, de transparentar a los estudiantes qué es lo que va a mirar de ellos cuando los vaya a visitar a sus clases. Algo así como una «rúbrica viva», como afirma la docente del caso 2 durante la entrevista.

\section{Discusión}

Cada caso se desarrolló con independencia y sin intervención del equipo de investigación. Un resultado posible era que alguno de ellos manifestara la imposibilidad de trabajar con los dispositivos, por diferentes razones: no adecuación de los dispositivos a la dinámica de clase, falta de adaptación de los estudiantes o docentes a esta modalidad propuesta, dificultades tecnológicas, entre otras. Sin embargo, esto no sucedió y se pudieron llevar adelante todas las actividades de aprendizaje planificadas por los docentes. De esta forma, se puede afirmar que el dispositivo valorado se adecua a una aceptable diversidad de perfiles de usuarios y contextos de uso.

Se abarcó un espectro de docentes de diferentes campos del conocimiento. Se trató de materias que presentan epistemologías, uso de recursos y estrategias didácticas diferentes. La diversidad de modalidades metodológicas que desarrollaron los docentes, desde diferentes disciplinas y en diferentes niveles de la formación pre profesional, estarían indicando una potencialidad de la innovación en cuanto a ajustarse a diferentes estilos de los formadores, así como a diversos grados de profundidad en el análisis didáctico. Además, permitiría adecuarse también a las opciones didácticas relacionadas con las epistemologías de las especialidades (Libaneo, 2010).

Vaillant (2019) afirma que la innovación debe tener la posibilidad de articularse/alternarse y sumarse a los procesos en marcha: ser disruptor no significa fragmentar. La innovación propuesta se sembró en un terreno fértil, predispuesto. La disponibilidad en Uruguay de conectividad es ubicua, 85\% de los hogares accede a banda ancha (Uruguay XXI, 2019). Todos los docentes de didáctica, así como los estudiantes en el último año de la carrera reciben una computadora portátil gratuita por el Plan Ceibal. También cabe destacar que todos los centros de formación docente del país poseen laboratorios de informática con computadoras fijas disponibles y portátiles para préstamo de los estudiantes. El 95,5\% de los estudiantes consideran que el uso de internet «es un medio importante como apoyo a las actividades académicas y curriculares» (CFE, 2015, p. 27).

Algunas investigaciones regionales contemporáneas destacan la importancia de la articulación de la formación inicial con el sistema de formación 
permanente de los docentes (Chávez y Vieira, 2018). Se han identificado en países latinoamericanos dificultades en ese sentido, lo que provoca perder la oportunidad de intervenir con continuidad, atendiendo emergentes, y contar con docentes que sientan que tienen la posibilidad de cumplir con la premisa de aprender toda la vida. Vaillant (2019) identifica como característica favorable de la innovación, que proponga dispositivos que puedan ser adoptados no solo en la formación inicial sino también permanente. En esta investigación las evidencias presentadas muestran que los estudiantes y docentes identifican los dispositivos como pasibles de ser utilizados en diversos niveles, no solo en la formación inicial sino también con docentes egresados.

La observación de videos en $360^{\circ}$ permite la formación de imágenes mentales, pero no la sensación de presencia (Bogicevic et al., 2019). Sin embargo, puede afirmarse a favor del material en $360^{\circ}$ producido en este proyecto, que los escenarios en los que se desarrolla la actividad educativa a ser analizada es real, no hipotética ni modelada. También corre a favor que el acceso a los materiales se logra desde un navegador común, y son descargables. Lo que no se ha logrado, es una buena calidad en la imagen y en el sonido, y eso ha sido destacado como limitación tanto por parte de los formadores como de los estudiantes de didáctica que participaron.

Las dificultades que encontraron algunos estudiantes y docentes para acceder desde la red pública a las unidades fílmicas, se habrían superado porque están actualmente disponibles desde un espacio formal del sistema de formación docente, lo cual mejora la eficacia del recurso. La posibilidad de que ellos puedan realizar grabaciones en $360^{\circ}$ de sus actividades de enseñanza y de sus compañeros, es aún un objetivo difícil por la necesidad de equipos especiales.

La innovación no puede ser solo una novedad, debe presentar profundidad en la propuesta, incluir la reflexión y el análisis. Deben estar presentes la colaboración, la claridad conceptual y buena estructuración con relación a los objetivos de aprendizaje, aprender desde la "clínica» (Vaillant, 2019, p. 2), con casos de la práctica que puedan ser analizados desde la teoría, y la reflexión. El factor determinante es la propuesta y no la calidad del recurso, aunque seguramente este puede actuar como factor limitante, en casos puntuales.

\section{Conclusiones}

Los dispositivos generados constituyen una herramienta de aprendizaje que adquiere valor más bien por la intervención didáctica que se realiza con ella 
y no tanto por la calidad técnica. La valoración de la innovación se llevó adelante en un campo que estaba favorablemente predispuesto.

La usabilidad de los dispositivos responde a algunos de los criterios presentados por De Fátima et al. (2020), tales como proporcionar más oportunidades de acceso al conocimiento, de forma democrática, accesible e inclusiva. Ello permite afirmar que se estaría cumpliendo uno de los aportes esperados del proyecto, diversificar y enriquecer la formación de docentes del país. Pero aún pueden y deben mejorarse la calidad de las unidades fílmicas y progresar hacia la realidad virtual.

La posibilidad de realizar filmaciones en $360^{\circ}$ es aún un paso a concretar en la generalidad de los institutos de formación docente, se invita a los colectivos a utilizar las unidades fílmicas disponibles en el repositorio abierto del proyecto (http://recyreap.cfe.edu.uy/), mientras se avanza al objetivo de incrementar esta base.

Desde esta investigación se apuesta a una innovación que no sea «silenciosa» (González y Cruzat, 2019, p. 111). La emergencia sanitaria que obligó a suspender las clases presenciales en marzo de 2020, coincidió con la culminación del presente proyecto; pocas semanas antes se había logrado disponer de las unidades fílmicas y los protocolos en un repositorio online abierto. Queda ahora investigar el impacto que tuvo el uso de estos materiales, alternativa disponible en un momento en que no se podía realizar la práctica pre profesional de forma presencial en ningún lugar del país.

El equipo de trabajo de esta investigación agradece a los docentes, estudiantes, familias, equipos de gestión y funcionarios de las instituciones educativas que dieron autorización y abrieron las puertas de las aulas para poder llevar adelante las filmaciones.

\section{REFERENCIAS BIBLIOGRÁFICAS}

Aguerrondo, I. (2014). Planificación educativa y complejidad: gestión de las reformas educativas. Cuadernos de Pesquisa, 44 153), 548-578. https://doi. org/10.1590/198053142910

Aguerrondo, I. y Tiramonti, G. (2016). El futuro ya llegó... pero no a la escuela argentina. ¿Qué nos atrasa y dónde está el futuro de nuestra educación? Buenos Aires: Proyecto Educar 2050. Recuperado de https://educar2050.org.ar/ wp/wp-content/uploads/2015/11/Paper-Innovaci\%C3\%B3n-2016.pdf

Anijovich, R. y Cappelletti, G. (2018). Despertar la reflexión en la formación docente: dispositivos efectivos. Innoeduca. International Journal of Technology and Educational Innovation, 4(1), 24-35. Recuperado de http:// 
www.revistas.uma.es/index.php/innoeduca/article/view/3610 https://doi. org/10.24310/innoeduca.2018.v4i1.3610

Bogicevic, V., Seo, S., Kandampully, J. A., Liu, S. Q., y Rudd, N. A. (2019). Virtual reality presence as a preamble of tourism experience: The role of mental imagery. Tourism Management, 74, 55-64. https://doi.org/10.1016/j. tourman.2019.02.009

CFE (2015). Los estudiantes de formación en educación. Estudio sobre datos aportados por el censo de estudiantes del cfe 2014-2015. Montevideo: CFE. Recuperado de http://www.cfe.edu.uy/images/stories/pdfs/documentos_ aprobados_cfe/estudio_censo_2014-2015.pdf

Chávez, Á. y Vieira, S. (2018). La formación de profesores en Perú y Brasil: aproximaciones y diferencias. Educación, 27(52), 26-45. https://doi. org/10.18800/educacion.201801.002

De Fátima, M., Pedreira da Silva, A. y Ferreira da Costa, L. (2020). Memória e curadoria digital de museu e patrimônio: Avaliação de usabilidade $360^{\circ}$. Prisma.Com, 41, 191-215. https://doi.org/10.21747/16463153/41a10

Fernández, J.P. (2016). La adquisición y desarrollo de la competencia digital en alumnos deeducación secundaria. Estudio de caso. Cuadernos de Investigación Educativa, 7(2). https://doi.org/10.18861/cied.2016.7.2.2612

Fernández, J., Elórtegui, N. y Medina, M. (2003). Los incidentes críticos en la Formación y Perfeccionamiento del Profesorado de Secundaria de Ciencias de la Naturaleza. Revista Interuniversitaria de Formación del Profesorado, 17(1), 101-112. Recuperado de: https://www.redalyc.org/ pdf/274/27417107.pdf

Fernández, T. y Fernández J. (1994). Técnica de trabajo con profesores sobre su práctica docente: "Terapia de Knoll». Investigación en la Escuela, 22, 91-103. Recuperado de: https://revistascientificas.us.es/index.php/IE/ article/view/8507/7585

González, C., y Cruzat, M. (2019). Innovación educativa: La experiencia de las carreras pedagógicas en la Universidad de Los Lagos, Chile. Educación, 28(55), 103-122. https://doi.org/10.18800/educacion.201902.005

Hernández, R., Fernández, C. y Baptista, P. (2010). Metodología de la investigación. México: Mc Graw-Hill.

Landín, M. del R. (2015). El Proyecto Aula. Una propuesta de innovación para la docencia y la formación profesional. Educación, 24(46), 117-131. Recuperado de http://revistas.pucp.edu.pe/index.php/educacion/article/ view/12247

Paz Sandín, M. (2003). Investigación cualitativa en educación. Fundamentos y tradiciones. Madrid: Mc Graw-Hill. 
Umpiérrez, S. y Rodríguez, E. (2017). Aportes para el diseño de una herramienta para el seguimiento y evaluación de experiencias innovadoras. Revista de Estudios y Experiencias en Educación, 16(31), 107-126. https://doi. org/10.21703/rexe.2017311071267

Uruguay XXI (18 de diciembre de 2019). Uruguay: una revolución tecnológica en poco más de una década. Recuperado de https://www.uruguayxxi.gub.uy/ es/noticias/articulo/uruguay-una-revolucion-tecnologica-en-poco-mas-deuna-decada/

Vaillant, D. (2019). Formación del profesorado para la innovación. En M. Martínez Martín y A. Jolonch i Anglada (Coods.), Las paradojas de la Innovación Educativa. Barcelona: Horsori. 